\title{
Double origin of the superior cerebellar artery associated with homolateral haemorrhagic infarction of cerebellum
}

\author{
Andrea Porzionato ${ }^{1}$, Veronica Macchi ${ }^{1}$, Luca Massaro ${ }^{1}$, Aldo Morra ${ }^{2}$, Gloria Sarasin ${ }^{1}$, Anna Rambaldo ${ }^{1}$, Raffaele De Caro ${ }^{1}$ \\ ${ }^{1}$ Institute of Human Anatomy, University of Padova, ${ }^{2}$ Euganea Medical Center, Padova, Italy
}

\begin{abstract}
The superior cerebellar artery (SCA) shows the least variable course and the lowest incidence of anatomical variations among cerebellar arteries. In the present study, an 84-year-old woman was affected by a cerebellar infarction which underwent haemorrhagic evolution in the following days. Neuroimaging investigations also showed a probable double origin of the left SCA. Neuropathological examination confirmed the presence of a large haemorrhagic infarction at the level of the superior portion of the left cerebellar hemisphere and vermis. The left SCA arose from two small arteries arising from the left aspect of the basilar artery and joining together after a course of $9 \mathrm{~mm}$. Previous studies have reported the association of cerebrovascular pathologies, such as intracranial aneurysms, with fenestrations and double origins of the posterior inferior cerebellar artery. In the present case, the occurrence of an haemorrhagic infarction in the vascular field of an SCA with double origin is intriguing in suggesting a possible pathophysiological association.
\end{abstract}

Key words: superior cerebellar artery, cerebellum, anatomical variation, stroke, xanthogranuloma, fenestration, angiography, magnetic resonance, computed tomography, neuroimaging.

\section{Introduction}

The superior cerebellar artery (SCA) is the least variant among cerebellar arteries, showing by far the lowest prevalence of anatomical variations. However, the incidence of double SCA in magnetic resonance angiographies has been reported to go from $3 \%$ to $10 \%[8,24,26,27]$ and reports of triple SCA are also present in the literature $[6,17,34]$. The SCA may also arise from the proximal segment of the posterior cerebral artery, a pattern corresponding to a joint origin of the two vessels $[24,27]$. Fenestration of the SCA has also been rarely described $[24,27]$ but to the best of our knowledge, angiographic or post-mortem reports of double origins of SCA are not available.
Thus, in this paper we present the first neuroradiological and neuro-pathological description of a double origin of SCA, which was also associated with haemorrhagic infarction of the corresponding cerebellar territory.

\section{Case description}

An 84-year-old woman underwent syncope with head trauma. A cerebral computed tomography (CT) performed in the local emergency department was negative, except for very small haematic hyperdensities in the cortex of the left insula and right parietal lobe. Neurological examination was also negative, apart from headache and one episode of vomiting. 
Cerebral CT was repeated the following day and, due to absence of significant changes, the woman was released. On these CTs, two calcified small masses, potentially ascribable to benign xanthogranulomas, were also visible at the level of the choroid plexuses of the lateral ventricles (Fig. 1).

In the following four weeks, the woman had progressive headache worsening and about after one month nausea and vomiting appeared again. Moreover, on the $35^{\text {th }}$ day of clinical course, dizziness and balance disorders appeared and on the $39^{\text {th }}$ day she was admitted again to hospital because of a fall without being able to get up. On admission, a cerebral CT showed large hypodensity in the left paravermian region of cerebellum, partially extending to the right paravermian region; in the context of the lesion, a hyperdense area was also present, which was ascribed to haemorrhagic infiltration (Fig. 2). A cerebral magnetic resonance $(M R)$ on the $43^{\text {rd }}$ day showed T2 flair hypersignal area in the territory of the left superior cerebellar artery, involving the homolateral middle cerebellar peduncle and the vermis; at the level of the superior vermis, a GE T2 hyposignal
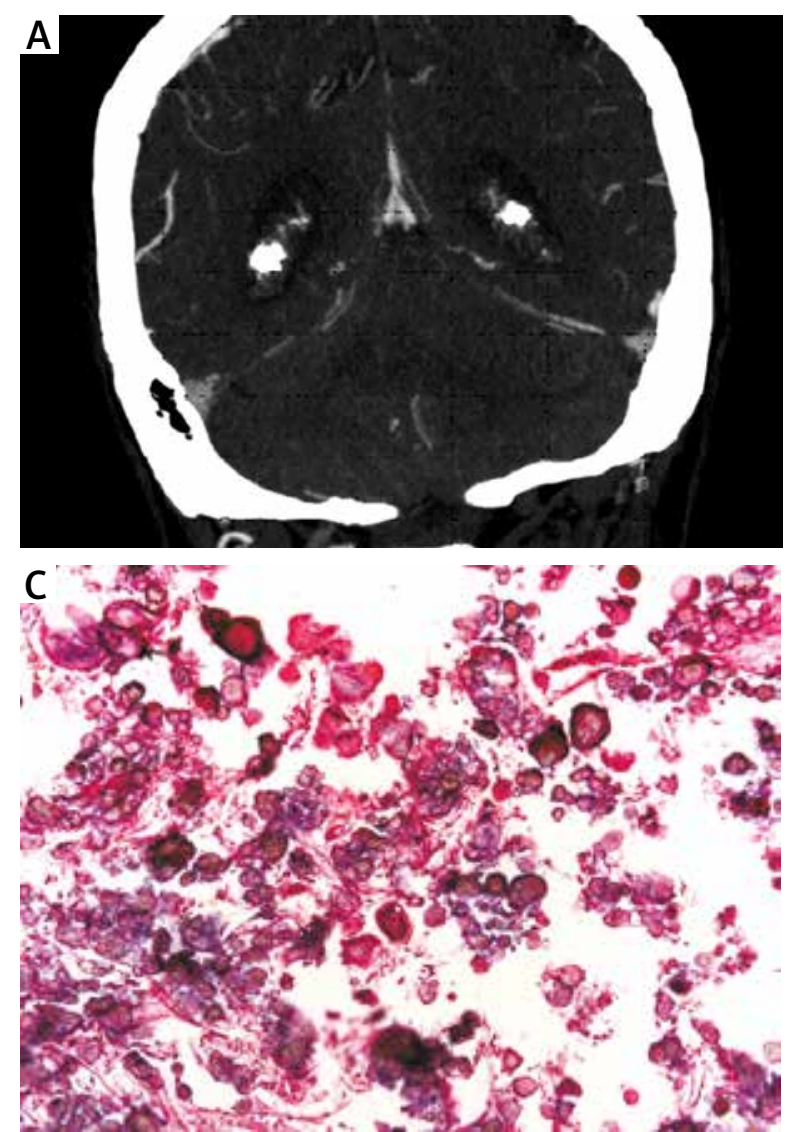

focus was also confirmed. Neuroradiologists made a diagnosis of subacute ischaemic lesion of the territory of the left superior cerebellar artery, with following modest haemorrhagic infarction. On the $46^{\text {th }}$ day after syncope, CT angiography did not show vessel occlusions but identified duplication of the origin of the left superior cerebellar artery (Fig. 3). Meanwhile, the neurologic conditions of the woman worsened, with dysarthria, dysmetria, and ataxia. On the $55^{\text {th }}$ day, a cerebral CT showed the evolution of the cerebellar lesion, which appeared as a voluminous hypodense area in the left cerebellum, with areas of intense contrast impregnation, partially imprinting the IV ventricle. The patient died on the $67^{\text {th }}$ day of her clinical course.

Neuropathological examination was performed after fixation in $10 \%$ formalin. Examination of the vessels of the cerebral base showed mild asymmetry in the calibre of the vertebral arteries, with the left and right arteries showing diameters of 3.5 and $2 \mathrm{~mm}$, respectively. The basilar artery had a diameter of $3.5 \mathrm{~mm}$. The left SCA arose from two small arteries arising from the left aspect of the basilar artery, at

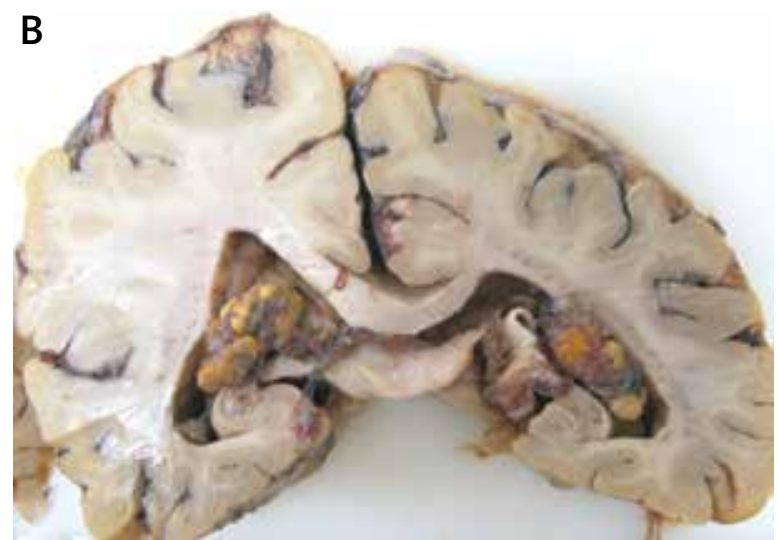

Fig. 1. Bilateral xanthogranuloma of the lateral ventricles at cerebral computed tomography (A), and macroscopic (B) and microscopic (C) examination. Note the several calcified concretions on the section stained with hematoxylin-eosin. 

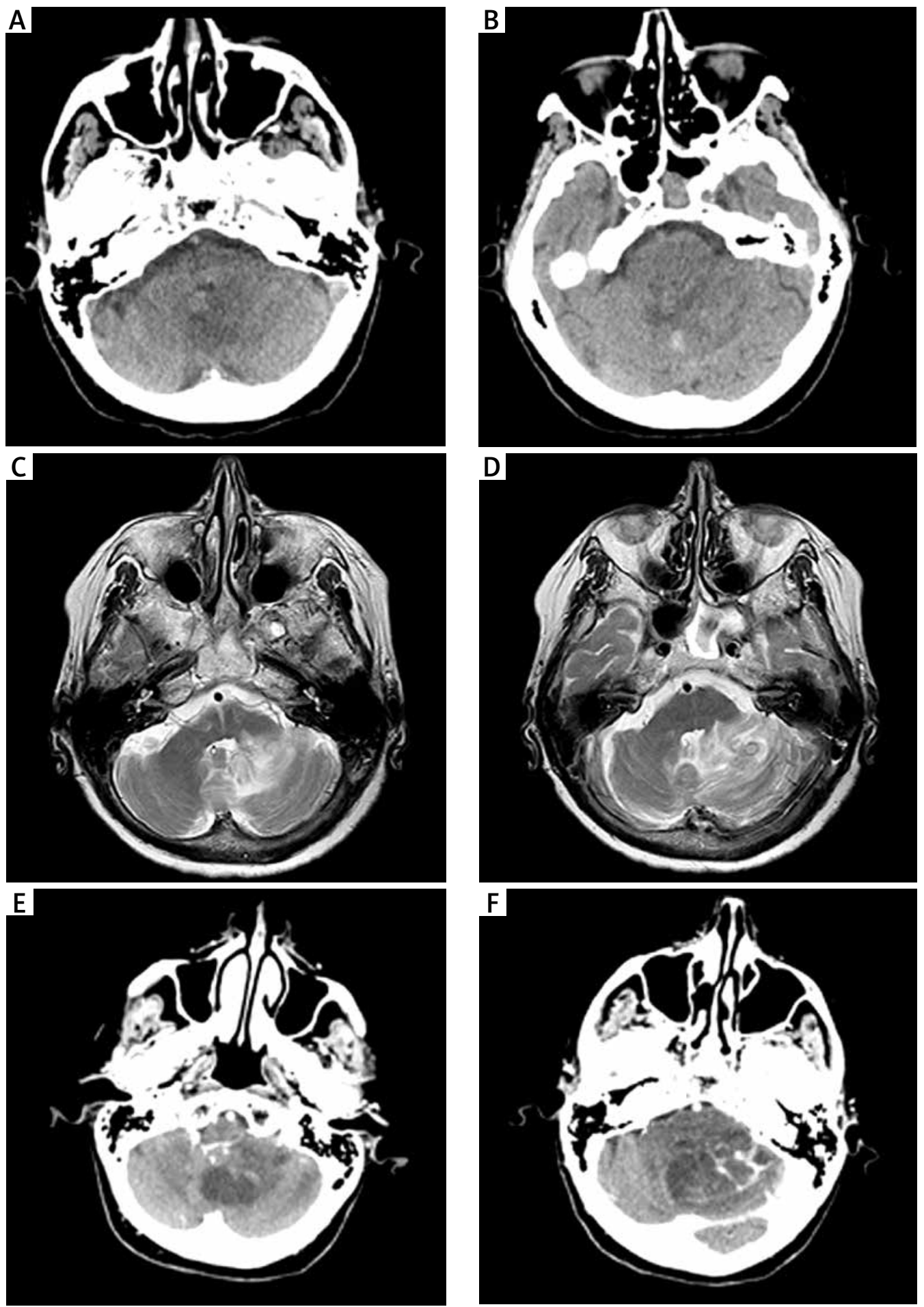

Fig. 2. A-B) Cerebral computed tomography (CT) on the $39^{\text {th }}$ day of clinical course. Note the large hypodensity in the left paravermian region, partially extending to the right side, with a hyperdense area in its context. C-D) Cerebral magnetic resonance imaging (MRI) on the $43^{\text {rd }}$ day showing T2 flair hyperintense area in the superior portions of the left cerebellar hemisphere, partially extending to the vermian and right paravermian region; a GE T2 hypointense area is also visible. E-F) Cerebral CT on the $55^{\text {th }}$ day, more clearly showing the cerebellar lesion as a voluminous hypodense area with foci of intense contrast impregnation. 
a reciprocal distance of $0.5 \mathrm{~mm}$, and joining together after a course of $9 \mathrm{~mm}$ (Fig. 4A-B). Each vessel had an external diameter of about $0.5 \mathrm{~mm}$ along all of the course and up to the confluence; then, the SCA arisen from the two vessels showed a diameter of about $1 \mathrm{~mm}$. A comparative analysis of the left SCA with the right SCA clearly showed that the two vessels giving the left SCA had smaller diameters than the right SCA, which showed a diameter never smaller than $1 \mathrm{~mm}$.

Brainstem, still connected with cerebellum, was separated from cerebrum through a transverse section at the level of the mesencephalon. Macroscopic examination of the cerebellum showed bulging of the superior surface of the left hemisphere, with swelling of the cerebellar folia and partial homogenization of the nervous tissue (Fig. 4C). On the inferior aspect of cerebellum, a sulcus of tonsillar herniation was appreciable. Brainstem and cerebellum were cut together with sections parallel to the cerebellar circumference. In the left cerebellar hemisphere, an irregularly ellipsoid cavity of $3 \times 2.5 \times 1.5 \mathrm{~cm}$, with necrotic-haemorrhagic content and anfractuous margins, was found. The lesion partially extended to the cerebellar vermis and the right paravermian region (Fig. 4D-E).

Section of the brain through multiple coronal planes showed two nodular lesions, with lobulated yellowish surfaces and firm consistence, at the choroid plexuses of the lateral ventricles (Fig. 1B).

Brainstem/cerebellum slices were paraffin embedded and sectioned. Histopathological examination confirmed the necrotic-haemorrhagic nature of the lesion (Fig. 4F) and revealed inflammatory leukocyte infiltrations of its walls. Thus, neuropathological
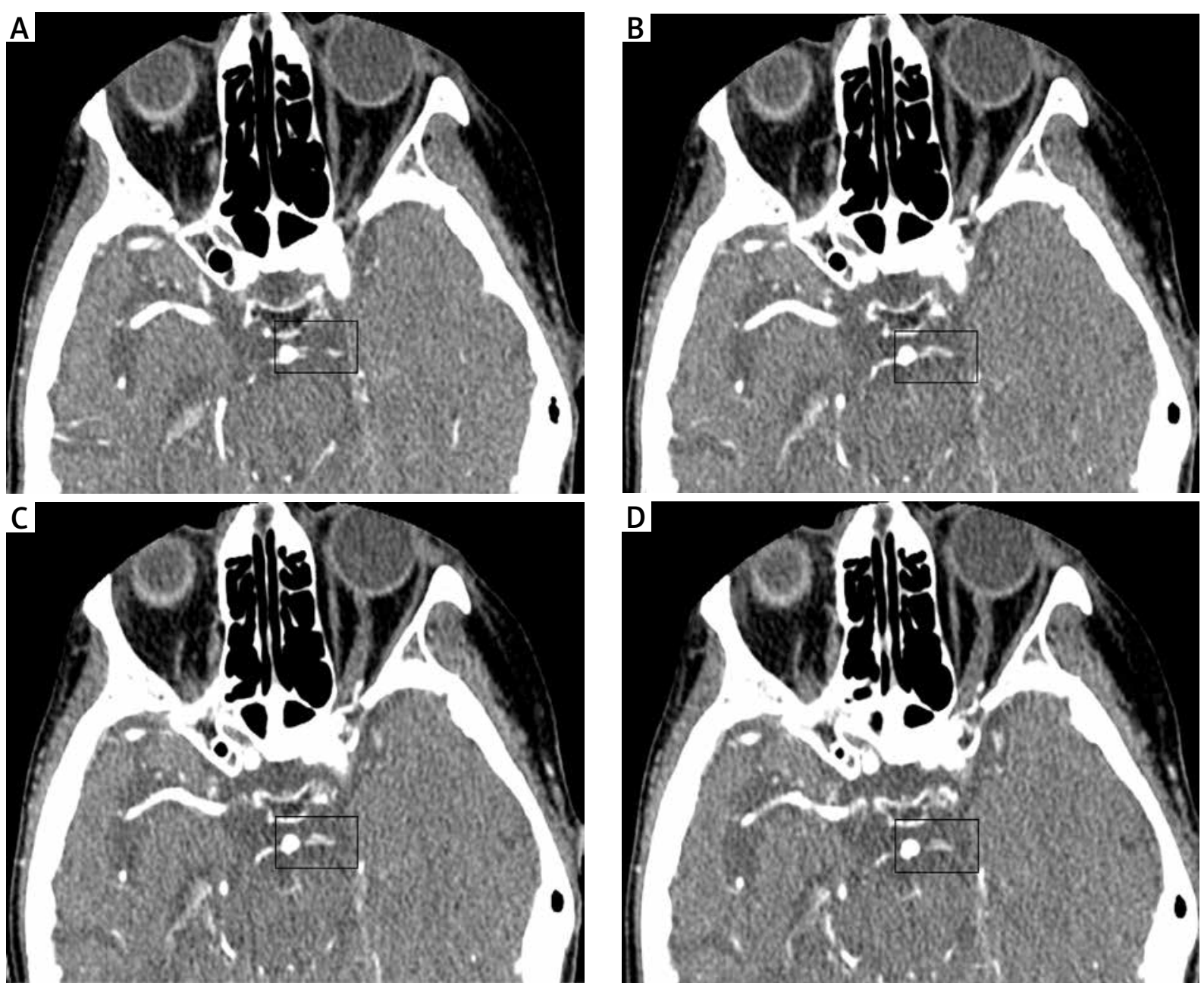

Fig. 3. Computed tomography angiography on the $46^{\text {th }}$ day showing the two components of the superior cerebellar artery arising separately from the vertebral artery and converging after brief courses into a single vessel. 

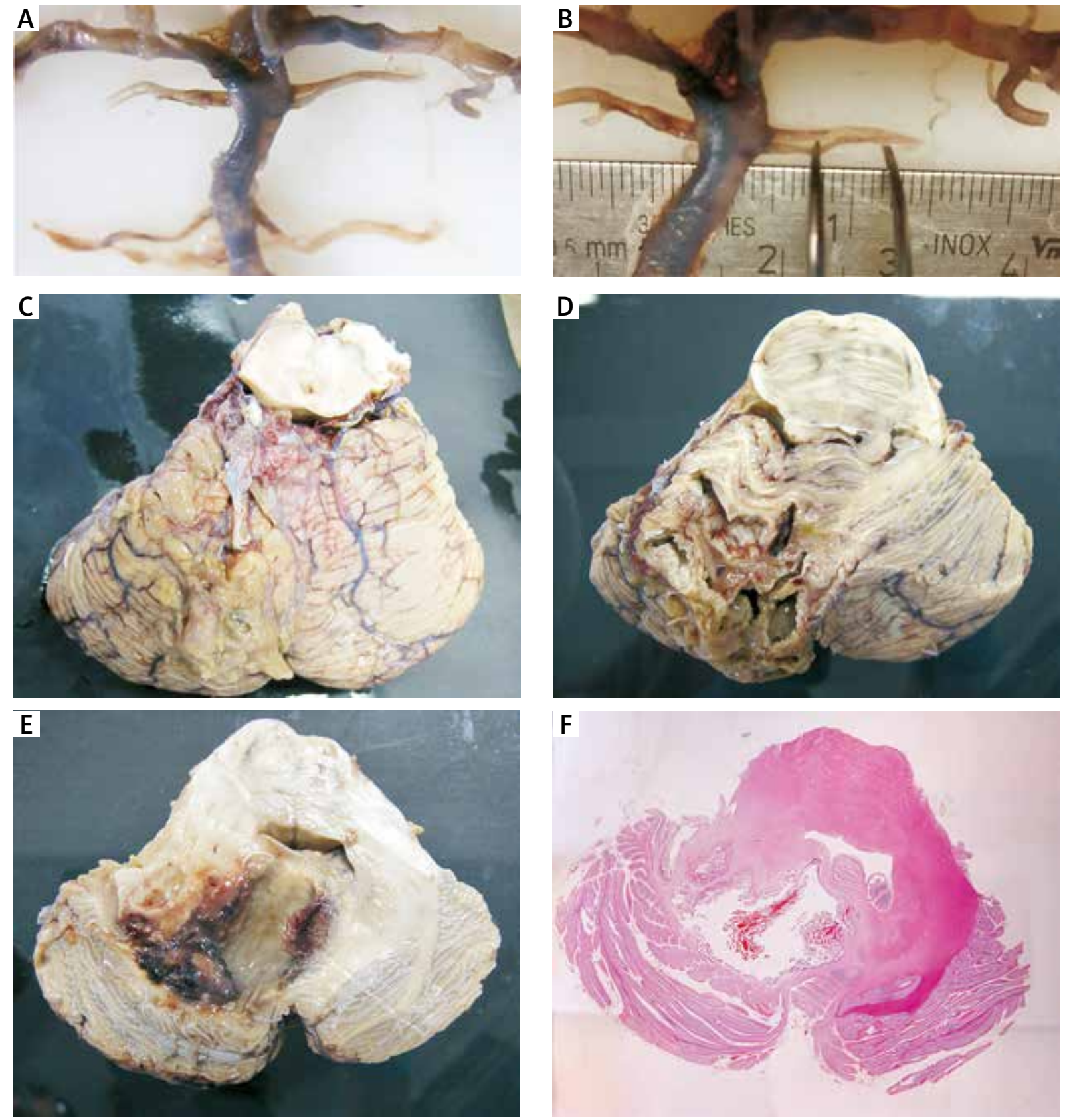

Fig. 4. A-B) Vision of the double origin of the superior cerebellar artery at macroscopic examination of the vessels of the brain base after their sampling. C) Superior surface of the cerebellum, showing bulging of the left cerebellar hemisphere, together with swelling and partial homogenization of the nervous tissue. D-E) Superior views of cerebellum after removing of the first and second slices in cranio-caudal progression. Note the large cavity with necrotic-haemorrhagic content and irregular margins. F) Cerebellar macro-section, corresponding to Figure $\mathrm{E}$, at the level of the ischaemic lesion.

examination confirmed the neuroradiological diagnosis of subacute ischaemic lesion with haemorrhagic evolution of the left SCA territory. As regards the nodular structures in the lateral ventricles, multiple cho- lesterol clefts and several calcified concretions (Fig. 1C) were found in a context of granulomatous tissue, confirming the diagnosis of bilateral xanthogranuloma as incidental finding. 


\section{Discussion}

To the best of our knowledge, this is the first report of double origin of SCA, which is usually considered the most constant cerebellar artery. The AICA also shows quite a low incidence of anatomical variations; fenestrations are very rare [28] and double origins of AICA are also not present in common databases of medical literature. The PICA, instead, is the most variable. PICAs with double origin have previously been reported in the literature both in angiography[1,3,10-12,14,15,19-21,29]andautopsy[22,32]. Fenestrations of the PICA have also been reported in few case reports $[16,23]$ and contemporary presence of double origin and fenestration of one of the two components has been reported only twice $[1,12]$.

The two components of a double origin of PICA usually arise at a certain distance to each other; Kwon et al. [10] have reported a distance between the two channels ranging from 19.5 to $35.6 \mathrm{~mm}$, with the mean value of $24.9 \mathrm{~mm}$. There are no descriptions or images of double origins of PICA with the two vessels at less than $1 \mathrm{~mm}$ to each other. Thus, the anatomical situation of the double origin of SCA described in the present paper is quite different, as in our case the two channels converging in the SCA arisen from the basilar artery at about $0.5 \mathrm{~mm}$ to each other.

The above difference between the two types of double origins may be a consequence of different morphogenetic mechanisms. The double origin of the PICA has been ascribed to persistence of anastomosis of PICA with the lateral spinal artery. In particular, the lateral spinal artery would become the caudal component of the double origin of the PICA while the rostral component would represent the PICA proper, embryologically derived from a hypertrophied radiculopial artery $[11,14,15,21]$. Some authors proposed that PICA fenestrations could result from partial regression of double origins [16]. Conversely, Lee et al. [12] have described the contemporary presence of double origin and fenestration as due to a hybrid of developmental variations, such as fenestration, duplication, abnormal regression and anomalous arterial origin. In the presented case of double origin of SCA, the abnormal persistence of an embryological vessel may also be hypothesized, although a very close relationship between the two vessels and the extreme rarity of the anatomical variation may not exclude alternative rarer mecha- nisms, such as for instance the division of an originally unique vessel.

Previous studies have reported the association of intracranial aneurysms with fenestrations and double origins of the posterior inferior cerebellar artery $[1,14,15]$. Aneurysms have been reported near the double origin of PICA or far away from the anatomical variation. In some cases, aneurysms have been specifically reported in the cranial or caudal channels of a double origin of PICA $[9,10,13,19,20,29]$ or in the PICA after convergence of the two channels [9]. In particular, it has been suggested that double origin of the PICA could represent the effect of an underlying disorganization of the vascular development, which increases the risk of acquired intracranial aneurysms [15]. Some authors also recommended that a thorough search for intracranial aneurysms should be performed if a double origin of PICA is detected [21].

Some authors have also reported that one or both components of a double origin of PICA may be used as routes for endovascular treatments. In the cases when aneurysms are located in one of the two components of the double origin, the affected channel may be sacrificed through endovascular trapping, permitting preservation of blood flow through the other channel $[10,13,19,20,29]$. Only in one reported case such an approach has caused medullary infarction, probably due to occlusion of the perforating vessel at the tonsillomedullary segment [20], but balloon occlusion test has also been recently proposed for evaluation of the safety of the procedure [13]. We can consider that in a double origin as the one we described, endovascular access to one of the channels would probably be very difficult due to small calibres and close proximity of the vessels.

Apart from aneurysms, a pial arteriovenous fistula has also been reported at the level of a double origin of PICA, supporting the hypothesis of alterations in vascular development as a common pathogenetic mechanism [5]. Moreover, it must be considered that fenestrations of intracranial arteries have also been associated with other vascular anomalies, such as persistence of embryonal vessels, moyamoya disease and arteriovenous malformations [7,25,30,33].

In our case, aneurysms (or other of the above anomalies) were not present in the arteries of the brain base; however, bilateral xanthogranuloma of the choroid plexuses of the lateral ventricles coexisted. They are benign idiopathic lesions which are 
usually asymptomatic and show an incidence of $1.6 \%$ to $7 \%$ of autopsies [18,31]. Their pathogenesis has not yet been clarified. Aging processes have been suggested, possibly correlated with hypercholesterolemia, atherosclerosis or diabetes mellitus, but an aberrant embryological development of the choroid plexus has also been proposed. In particular, xanthogranuloma may originally derive from neuroepithelial tubules (endowed of increased proliferative capacity) abnormally displaced in the stroma of choroid plexus during epithelial invagination [4]. Thus, also in our case, the coexistence of double origin of a cerebellar artery with another neuropathological entity possibly due to anomalous embryologic maturation of cerebral vascularization suggests the involvement of underlying developmental mechanisms.

Cerebellar haemorrhages may have particular characteristics with respect to other cerebrovascular districts. For instance, cerebellar small bleeds have been reported with higher frequency in cerebral micro-angiopathy due to hypertension and in progressive supranuclear palsy [2]. In the present case, the occurrence of a haemorrhagic infarction in the vascular field of an SCA with double origin is intriguing in suggesting a possible pathophysiological association. Although associated with a long-term compensation, the double origin of the left SCA may have represented an anatomic situation of haemodynamic instability, also due to the small distance between the two origins and the small diameters of the two vessels. Neuro-radiological and neuro-pathological investigations did not identify significant atherosclerotic disease nor possible sources of embolisms. Thus, it may be hypothesized that a hypotensive episode may have caused ischaemic damage to the territory of the left SCA, made more vulnerable due to the particular haemodynamic situation given by the double origin of the left SCA.

\section{Disclosure}

Authors report no conflict of interest.

\section{References}

1. Cho YD, Han MH, Lee JY. Double origin of the posterior inferior cerebellar artery with juxta-proximal fenestration of caudal component. Surg Radiol Anat 2011; 33: 271-273.

2. De Reuck J, Caparros-Lefebvre D, Deramecourt V, Defebvre L, Auger F, Durieux N, Bordet R, Pasquier F, Maurage CA. Prevalence of small cerebral bleeds in patients with progressive supranucle- ar palsy: a neuropathological study with 7.0-Tesla magnetic resonance imaging correlates. Folia Neuropathol 2014; 52: 421-427.

3. Fan F, Wang C, Xie X. Endovascular treatment of a ruptured vertebral dissecting aneurysm associated with double-origin posterior inferior cerebellar artery. Catheter Cardiovasc Interv 2011; 77: 150-153.

4. Gaskill SJ, Saldivar V, Rutman J, Marlin AE. Giant bilateral xanthogranulomas in a child: case report. Neurosurgery 1992; 31: 114-117.

5. Guimaraens L, Casasco A, Sola T, Cuellar H, Miralbes S, Cambra FJ. Endovascular treatment of a pial arteriovenous fistula of a posteroinferior cerebellar artery with a double origin. J Neurointerv Surg 2011; 3: 233-236.

6. Hardy DG, Rhoton AL Jr. Microsurgical relationships of the superior cerebellar artery and the trigeminal nerve. J Neurosurg 1978; 49: 669-678.

7. Hattori T, Inoue S, Sakai N. Fenestration of the basilar artery associated with persistent primitive trigeminal artery. Neurol Med Chir 1997; 37: 841-843.

8. Hoksbergen AW, Majoie CB, Hulsmans FJ, Legemate DA. Assessment of the collateral function of the circle of Willis: threedimensional time-of-flight MR angiography compared with transcranial color-coded duplex sonography. AJNR Am J Neuroradiol 2003; 24: 456-462.

9. Koh JS, Lee CY, Lee SH, Kim GK. Dissecting aneurysm associated with a double origin of the posterior inferior cerebellar artery causing subarachnoid hemorrhage. J Korean Neurosurg Soc 2012; 51: 40-43.

10. Kwon BJ, Jung C, Im SH, Lee DH, Han MH. Double origin of the posteroinferior cerebellar artery: angiographic anatomy and endovascular treatment of concurrent vertebrobasilar dissection. Neurosurgery 2007; 61: 242-248.

11. Lasjaunias P, Vallee B, Person H, Ter Brugge K, Chiu M. The lateral spinal artery of the upper cervical spinal cord. Anatomy, normal variations, and angiographic aspects. J Neurosurg 1985; 63: 235-241.

12. Lee SH, Koh JS, Ryu CW, Bang JS. Fenestration of the double origin of the posterior inferior cerebellar artery associated with a contralateral vertebral artery dissection. Cerebellum 2009; 8: 382-384.

13. Lee SH, Koh JS, Ryu CW, Lee CY, Lee SJ. Successful endosaccular coiling after a balloon occlusion test of the caudal channel of a double origin of the posterior inferior cerebellar artery originating from the aneurysm neck. Interv Neuroradiol 2011; 17: 183-187.

14. Lesley WS, Dalsania HJ. Double origin of the posterior inferior cerebellar artery. AJNR Am J Neuroradiol 2004; 25: 425-427.

15. Lesley WS, Rajab MH, Case RS. Double origin of the posterior inferior cerebellar artery: association with intracranial aneurysm on catheter angiography. AJR Am J Roentgenol 2007; 189: 893-897.

16. Lesley WS. Fenestration of the posterior inferior cerebellar artery. Cerebellum 2008; 7 : 240-241.

17. Mani RL, Newton TH, Glickman MG. The superior cerebellar artery: an anatomic-roentgenographic correlation. Radiology 1968; 91: 1102-1108.

18. Moreau E, Lefrancq T, Saint-Martin P. Incidental bilateral xanthogranuloma of the lateral ventricles at autopsy - a case report. J Forensic Leg Med 2013; 20: 647-649. 
19. Padovani Trivelato F, Salles Rezende MT, Brito Santos R, Hilton Vieira Madeira T, Cardoso Campos R, Cordeiro Ulhôa A. Intracranial aneurysms associated with a double origin of the posterior inferior cerebellar artery. Interv Neuroradiol 2011; 17: 351-356.

20. Pasco A, Thouveny F, Papon X, Tanguy JY, Mercier P, Caron-Poitreau C, Herbreteau D. Ruptured aneurysm on a double origin of the posterior inferior cerebellar artery: a pathological entity in an anatomical variation. Report of two cases and review of the literature. J Neurosurg 2002; 96: 127-131.

21. Plumb AA, Herwadkar A, Laitt R. Double origin of the posterior inferior cerebellar artery with findings on conventional and CT angiography. Surg Radiol Anat 2009; 31: 393-395.

22. Stopford JS. The arteries of the pons and medulla oblongata. J Anat 1916; 50: 130-164.

23. Theodosopoulos PV, Lawton MT. Fenestration of the posteroinferior cerebellar artery: case report. Neurosurgery 2000; 47: 463 465.

24. Tolabin I, Bertona CA, Bertona JJ, Gribaudo N. Anatomical variations of the circle of Willis in magnetic resonance angiography. Imagenes 2014; 3: 17-27.

25. Tran-Dinh HD, Dorsch NW, Soo YS. Ectasia and fenestration of the anterior cerebral artery associated with persistent trigeminal artery: case report. Neurosurgery 1992; 31: 125-128.

26. Uchino A, Sawada A, Takase Y, Kudo S. Variations of the superior cerebellar artery: MR angiographic demonstration. Radiat Med 2003; 21: 235-238.

27. Uchino A. Cerebral arterial variations and anomalies diagnosed by MR angiography. In: Neurovascular Imaging. Takahashi S (ed.) Springer-Verlag, London 2010

28. van Rooij SB, Bechan RS, Peluso JP, Sluzewski M, van Rooij WJ. Fenestrations of intracranial arteries. AJNR Am J Neuroradio 2015; 36: 1167-1170.

29. Vora N, Thomas AJ, Horowitz MB, Jovin T. Retrograde back-coiling technique for a ruptured aneurysm of a double-origin posterior inferior cerebellar artery. J Neuroimaging 2009; 19: 65-67.

30. Vörös E, Kiss M, Hankó J, Nagy E. Moyamoya with arterial anomalies: relevance to pathogenesis. Neuroradiology 1997; 39: 852 856.

31. Wolf A, Cowen D, Graham S. Xanthomas of the choroid plexus in man. J Neuropathol Exp Neurol 1950; 9: 286-297.

32. Yasargil MG. Microneurosurgery. Vol 1. Thieme Stratton, New York 1984.

33. Yoshimoto H, Maeda H, Aoyama H, Kanazawa J, Kitaoka T, Uozumi T. Enlargement of cerebellar arteriovenous malformation associated with fenestration of the vertebral artery - case report. Neurol Med Chir 1992; 32: 585-588.

34. Zenteno M, Moscote-Salazaar LR, Alvis-Miranda HR, Rojas A, Lee A. Unilateral triplication of superior cerebellar artery associated with fetal posterior cerebral artery: case report. Acta Medica Int 2015; 2: 158-159. 